\title{
Protogenetic sulfide inclusions in diamonds date the diamond formation event using Re-Os isotopes
}

\author{
Pamato M.G. ${ }^{1 *}$, Novella D. ${ }^{1,2}$, Jacob D.E. ${ }^{3}$, Oliveira B. ${ }^{4}$, Pearson D.G. ${ }^{5}$, Greene S. $^{4}$, Afonso \\ J.C. ${ }^{4}$, Favero M. ${ }^{1}$, Stachel T. ${ }^{5}$, M. Alvaro ${ }^{6}$, Nestola F. ${ }^{1}$ \\ ${ }^{1}$ Department of Geosciences, University of Padova, Via Gradenigo 6, 35131 Padua, Italy \\ ${ }^{2}$ Department of Biological, Geological and Environmental Sciences, University of Bologna, 40126 \\ Bologna, Italy \\ ${ }^{3}$ Research School of Earth Sciences, The Australian National University, Canberra ACT 2600, \\ Australia \\ ${ }^{4}$ Department of Earth and Environmental Sciences, Macquarie University, North Ryde, NSW 2109, \\ Australia \\ ${ }^{5}$ Department of Earth and Atmospheric Sciences, University of Alberta, Edmonton, Alberta T6G \\ 2E9, Canada \\ ${ }^{6}$ Department of Earth and Environmental Sciences, University of Pavia, 27100 Pavia, Italy \\ *corresponding author: martha.pamato@unipd.it
}

\section{Rietveld refinements and composition of the Mss}

It is generally agreed that sulfide inclusions encapsulated at the pressure and temperature conditions of diamond formation represent the original Mss in the Fe-Ni-S system (with 1-2\% $\mathrm{Cu}$ ) (Taylor and Liu, 2009). However, during cooling, the Mss exsolves to an intergrowth of distinct phases that fractionate the Re-Os system (Ballhaus et al., 2006), namely pyrrhotite [ $\left.\mathrm{Fe}_{1-x} \mathrm{~S}\right]$, pentlandite $\left[(\mathrm{Fe}, \mathrm{Ni})_{9} \mathrm{~S}_{8}\right]$, chalcopyrite $\left[\mathrm{CuFeS}_{2}\right]$ and occasionally pyrite $\left[\mathrm{FeS}_{2}\right]$. Molybdenite $\left[\mathrm{MoS}_{2}\right]$ and mackinawite $\left[(\mathrm{Fe}, \mathrm{Ni}){ }_{1+x} \mathrm{~S}\right]$ have also been recently identified in diamonds (Agrosì et al., 2017; Kemppinen et al., 2017). Thus, an assemblage of exsolved sulfide minerals is commonly encountered within diamonds, including in this study.

The occurrence of these three (pyrrhotite, pentlandite and chacolpyrite) compositionally distinct phases complicates the quantitative reconstruction of the original Mss inclusion. This problem is more severe for Ni-rich sulfides in peridotitic lithotypes than for Ni-poor sulfides in eclogites, if up to $5 \% \mathrm{Ni}$ is accomodated in pentlandite (Cabri, 1973). In fact, it is critical for isotopic analyses that 
the entire mass of the inclusion is analysed to ensure the quantitative reconstruction of the composition of the original bulk sulfide, given their differing Re-Os compositions (Taylor and Liu, 2009; McDonald et al., 2017). Inclusions in diamonds are commonly studied by destructive methods in which the diamonds are crushed or ablated using a laser. However, it is impossible to guarantee $100 \%$ recovery of the inclusion, and for laser ablation analyses partial sampling is the default situation introducing serious bias to geochemical results (McDonald et al., 2017).

The mineralogy and crystallography of sulfides has been extensively investigated (e.g. Kullerud, 1963; Craig, 1973; Wang and Salveson, 2005; Fleet, 2006) but, at present, very few X-ray diffraction measurements (Agrosì et al., 2017) fully identified the sulfides still entrapped in the diamond host. X-ray diffraction in-situ analyses are capable of quantifying the whole inclusion without compromising it via polishing, i.e., it provides a true "bulk" analysis. In fact, examination of polished sections to reconstruct the bulk composition of the sulfide results in higher uncertainties, as separate microcrystals are non-uniformly distributed and fractioned isotopes can be lost during sampling (Taylor and Liu, 2009; McDonald et al., 2017). Here, X-ray diffraction data were collected on single crystals and converted into a 2D powder patterns to perform Rietveld refinements of the diffraction pattern of the polycrystalline assemblage. This approach offers an alternative way to quantifying the relative amount of each sulfide phase present in the analysed volume.

The fundamental parameters approach (Cheary et al., 2004) as implemented in Profex-BGMN v.4.1.0 (Doebelin and Kleeberg, 2015) was used in the Rietveld refinements. Instrumental contribution to line broadening has been preliminary checked by fitting the diffraction profile of the LaB6 powder standard (NIST SRM-660b). This procedure involved the verification of the FPA configuration bundled with the software, after considering the single-crystal diffractometer setup: $120 \mu \mathrm{m}$ spot size, $177 \mathrm{~mm}$ goniometer radius and $0.172 \mathrm{~mm}$ detector pixel $1 \mathrm{D}$ dimension. Qualitative phase analysis was performed using PANalytical High Score Plus v.4.8.0 (Malvern Panalytical Ltd, Malvern, UK). Crystal structures of Pyrrhotite 5C (98-019-0010) and Diamond 
(98-005-3779) were taken from the ICSD structure database; Pentlandite (96-900-6138) and Chalcopyrite (96-101-0930) from the COD database. The refinement strategy included the variation of the following parameters: coefficients of the Lagrangian polynomial function used to describe the background ( 8 coefficients for each sample), scale factors, unit cell parameters, crystallite size, texture and microstrain. Neither atomic coordinates nor atomic displacement parameters were refined.

From the relative abundances of each sulfide phase obtained from the refinements and the chemical formulae of the individual endmembers $\left(\mathrm{Fe}_{9} \mathrm{~S}_{10}\right.$ for pyrrhotite $5 \mathrm{C} ; \mathrm{Fe}_{4.12} \mathrm{Ni}_{4.88} \mathrm{~S} 8$ for pentlandite and $\mathrm{CuFeS}_{2}$ for chalcopyrite), the original composition of the Mss was derived (Table DR1). The derived composition of the sulfides (Figure DR1) fall at the low-Ni end of the field of Mss with several inclusion overlapping the compositional field of pyrrhotite, in agreement with the compositions measured for eclogitic sulfides from Victor (Stachel et al., 2018). The derived average composition is $\mathrm{Fe}_{0.84(3)} \mathrm{Ni} 0.07(4) \mathrm{Cu} 0.02(1) \mathrm{S}_{1.0}$.

\section{Reciprocal Crystallographic orientations}

Reciprocal crystallographic orientations between sulfide inclusions and diamond hosts were calculated using the dedicated software OrientXplot (Angel et al., 2015, http://www.rossangel.com/textorientxplot.htm). As the main part of the inclusions consists of pyrrhotite single crystals and only very small amounts of other phases are exsolved (see Table DR1), the crystallographic relationships determined reflect the orientation of the original inclusion which do not show any phase transition. Pyrrhotite shows different and complex polytypes at room temperature and pressure (all based on a hexagonal NiAs type sub-cell), but the crystallographic details of the different polytypes will not be discussed here since this goes beyond the aim of this paper. For simplicity, the unit cell parameters and orientation matrices (Tables DR2-DR3) used in this study refer to the NiAs sub-cell (with $a=3.45 \AA$ and $c=5.75 \AA$ ). 
In the stereographic projections, one unambiguous orientation was chosen based on symmetry criteria, and the $a$ axis of the sulfides (purple dots) was plotted in a specific asymmetric unit (grey area), the $b$ axis (green dots) points upward, and the $c$ axis (blue dots) is constrained for a righthanded system. Empty symbols are directions plotting in the lower hemisphere. The relevant crystallographic data are reported in Tables DR1-DR2.

Sulfide inclusions in diamond from the Superior craton form an age array either due to coprecipitation of diamond and sulfide, or due to recrystallisation of sulfide during interaction with the diamond-forming fluid (Aulbach et al. 2018). As most of the sulfide inclusions investigated in this study are protogenetic, the hypothesis of co-precipitation is probably not valid. On the other hand, any metasomatic event large enough to produce a suite of sulfide-bearing diamond xenocrystals to fall on a Re-Os isochron should involve high fluid- or melt-rock ratios to induce recrystallisation. In this study, the presence of single crystals rather than polycrystalline phases does not indicate recrystallization processes. However whether dissolution-recrystallization rather than solid state recrystallization occurs cannot be ruled out from the crystallographic relationships between sulfides and their hosts. Nevertheless, if that is the case, the diffusion calculated are maximum timescales and in almost all cases, we would expect the Re-Os clock to be reset.

\section{Os diffusion model}

We consider that Os re-equilibration within sulfides depends on diffusive mass-flux through the mineral/liquid interface exclusively (i.e. no melting/crystallization reactions). This is modelled assuming that (a) the grain-boundary is always in equilibrium with the surrounding liquid (i.e. the Os concentration in the liquid and solid are linked by the partition coefficient) and (b) diffusion within the solid sulfide is obtained solving the following 1D differential equation:

$\frac{\partial C_{s}(x, t)}{\partial t}=D \frac{\partial^{2} C_{s}(x, t)}{\partial x^{2}}, \quad 0 \leq x \leq R$ 
where $C_{s}(x, t)$ is the concentration of Os along the $x$-direction of the sulfide ( $s$ refers to the solid sulfide), which is assumed to have slab symmetry. $R$ is the representative size of the mineral in the $x$ direction, which ranges from $\sim 100-300 \mu$ m. Other mineral shapes (e.g. cylindrical, spherical) result in faster diffusional equilibration. If $K$ is the equilibrium partition coefficient between the mineral and the liquid, then $(R, t)=K C_{1}$ is the concentration of the sulfide at its boundary, where $C_{1}$ is the concentration of Os in the liquid (assumed to be constant in an open system). Diffusion coefficients (D) for sulfides follow the Arrhenius equation from Brenan et al. (2000).

Equation (1) is solved using the built-in PDEPE function in MATLAB. The experiment is run until the concentration profiles reach the required equilibration level, defined as:

$e q=\left|\frac{i n t_{t=\infty}-i n t_{t}}{i n t_{t=\infty}-i n t_{t=0}}\right|, \quad$ int $t_{t}=\int_{0}^{R} C(x, t) d x$

Equilibrium is assumed when $e q=0.1$ and 0.001 , or in other words, when the mass of Os in the sulfide is $90 \%$ (solid lines in Fig. 4) and 99.9\% (dashed lines) in equilibrium with the surrounding liquid. According to Eqs. 1 and 2 the time for re-equilibration will depend on the size of the sulfide, and the diffusion coefficient.

\section{References}

Agrosì, G., Tempesta, G., Mele, D., Allegretta, I., Terzano, R., Shirey, S.B., Pearson, G.D., and Nestola, F., 2017, Non-destructive, multi-method, internal analysis of multiple inclusions in a single diamond: First occurrence of mackinawite $(\mathrm{Fe}, \mathrm{Ni}) 1+\mathrm{x}$ S: American Mineralogist, $\mathrm{v}$. 102, p. 2235-2243, doi:10.2138/am-2017-6178.

Angel, R., Milani, S., Alvaro, M., and Nestola, F., 2015, OrientXplot: A program to analyse and display relative crystal orientations: Journal of Applied Crystallography, v. 48, p. 1330-1334, doi:10.1107/S160057671501167X.

Ballhaus, C., Bockrath, C., Wohlgemuth-Ueberwasser, C., Laurenz, V., and Berndt, J., 2006, Fractionation of the noble metals by physical processes: Contributions to Mineralogy and Petrology, v. 152, p. 667-684, doi:10.1007/s00410-006-0126-z.

Brenan, J.M., Cherniak, D.J., and Rose, L.A., 2000, Diffusion of osmium in pyrrhotite and pyrite: Implications for closure of the Re-Os isotopic system: Earth and Planetary Science Letters, v. 180, p. 399-413, doi:10.1016/S0012-821X(00)00165-5.

Cabri, L.J., 1973, New data on phase relations in the Cu-Fe-S system, in Economic Geology, 
doi:10.2113/gsecongeo.68.4.443.

Cheary, R.W., Coelho, A.A., and Cline, J.P., 2004, Fundamental parameters line profile fitting in laboratory diffractometers, in Journal of Research of the National Institute of Standards and Technology, p. 1-25, doi:10.6028/jres.109.002.

Craig, J.R., 1973, Pyrite-Pentalndite assemblages and other low temperature relations in the Fe-NiS system: American Journal of Science, v. 273, p. 496-510.

Deines, P., and Harris, J.W., 1995, Sulfide inclusion chemistry and carbon isotopes of African diamonds: Geochimica et Cosmochimica Acta, v. 59, p. 3173-3188, doi:10.1016/00167037(95)00205-E.

Doebelin, N., and Kleeberg, R., 2015, Profex: A graphical user interface for the Rietveld refinement program BGMN: Journal of Applied Crystallography, v. 48, p. 1573-1580, doi:10.1107/S1600576715014685.

Fleet, M.E., 2006, Phase equilibria at high temperatures, in Reviews in Mineralogy and Geochemistry, doi:10.2138/rmg.2006.61.7.

Kemppinen, L.I.M., Kohn, S.C., Parkinson, I.J., Bulanova, G.P., Howell, D.H., and Smith, C.B., 2017, First identification of molybdenite in diamond-hosted sulphide inclusions and possible implications for Re-Os dating of diamonds: Earth and Planetary Science Letters, v. 495, p. 13, doi:10.1016/j.eps1.2018.04.037.

Kullerud, G., 1963, Thermal Stability Of Pentlandite: The Canadian Mineralogist, v. 7(3), p. 353.

McDonald, I., Hughes, H.S.R., Butler, I.B., Harris, J.W., and Muir, D., 2017, Homogenisation of sulphide inclusions within diamonds: A new approach to diamond inclusion geochemistry: Geochimica et Cosmochimica Acta, v. 216, p. 335-357, doi:10.1016/j.gca.2017.04.039.

Stachel, T., Banas, A., Aulbach, S., Smit, K.V., Wescott, P., Chinn, I.L., and Kong, J., 2018, The Victor Mine (Superior Craton, Canada): Neoproterozoic lherzolitic diamonds from a thermally-modified cratonic root: Mineralogy and Petrology, v. 112, p. 325-336, doi:10.1007/s00710-018-0574-y.

Taylor, L.A., and Liu, Y., 2009, Sulfide inclusions in diamonds: not monosulfide solid solution: Russian Geology and Geophysics, v. 50, p. 1201-1211, doi:10.1016/j.rgg.2009.11.018.

Wang, H., and Salveson, I., 2005, A review on the mineral chemistry of the non-stoichiometric iron sulphide, Fe $1-\mathrm{x} \mathrm{S}(0 \leq \mathrm{x} \leq 0.125)$ : polymorphs, phase relations and transitions, electronic and magnetic structures: Phase Transitions, v. 78, p. 547-567, doi:10.1080/01411590500185542. 


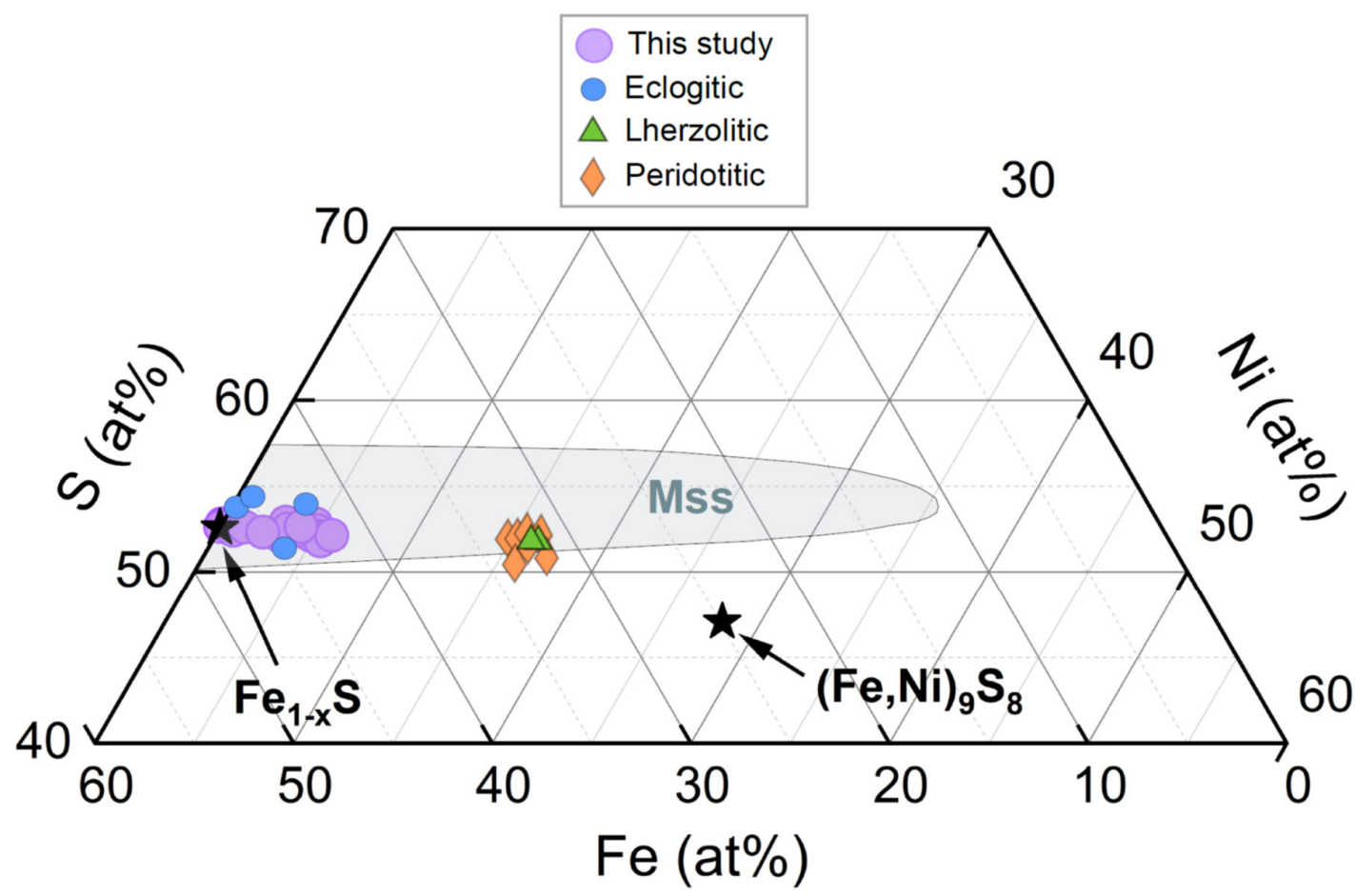

Figure S1: Composition (at\%) of sulfide inclusions from Victor mine in a Fe-Ni-S quadrilateral from this study. Eclogitic, lherzolitic and peridotitic sulfide inclusions from Victor from Stachel et al., (2018) are shown for comparison. Compositions of pyrrhotite and pentlandite are also shown (black stars). The field of the monosulfide solid solution (Mss) experimentally determined field for $1000{ }^{\circ} \mathrm{C}, 1 \mathrm{~atm}$ is shown in light grey (after Deines and Harris, 1995). 
Table S1: phase proportions of sulfide phases and derived Mss for Victor Mine diamonds

\begin{tabular}{|c|c|c|}
\hline Sample & Phase & Phase proportions (wt $\%$ ) \\
\hline \multirow[t]{4}{*}{ V2-01_inc_1 } & chalcopyrite & 3 \\
\hline & pentlandite & 1 \\
\hline & pyrrhotite & 96 \\
\hline & Mss composition & $\mathrm{Fe}_{0.88} \mathrm{Ni}_{0.01} \mathrm{Cu}_{0.01} \mathrm{~S}_{1.0}$ \\
\hline \multirow[t]{4}{*}{ V2-01_inc_2 } & chalcopyrite & 2 \\
\hline & pentlandite & 0 \\
\hline & pyrrhotite & 98 \\
\hline & Mss composition & $\mathrm{Fe}_{0.89} \mathrm{Cu}_{0.01} \mathrm{~S}_{1.0}$ \\
\hline \multirow[t]{4}{*}{ V2-01_inc_3 } & chalcopyrite & 1 \\
\hline & pentlandite & 0 \\
\hline & pyrrhotite & 99 \\
\hline & Mss composition & $\mathrm{Fe}_{0.90} \mathrm{Cu}_{0.003} \mathrm{~S}_{1.0}$ \\
\hline \multirow[t]{4}{*}{ V2-02_inc_1 } & chalcopyrite & 0 \\
\hline & pentlandite & 3 \\
\hline & pyrrhotite & 97 \\
\hline & Mss composition & $\mathrm{Fe}_{0.89} \mathrm{Ni}_{0.02} \mathrm{~S}_{1.0}$ \\
\hline \multirow[t]{4}{*}{ V2-02_inc_2 } & chalcopyrite & 3 \\
\hline & pentlandite & 4 \\
\hline & pyrrhotite & 93 \\
\hline & Mss composition & $\mathrm{Fe}_{0.87} \mathrm{Ni}_{0.03} \mathrm{Cu}_{0.01} \mathrm{~S}_{1.0}$ \\
\hline \multirow[t]{4}{*}{ V2-02_inc_3 } & chalcopyrite & 11 \\
\hline & pentlandite & 16 \\
\hline & pyrrhotite & 73 \\
\hline & Mss composition & $\mathrm{Fe}_{0.79} \mathrm{Ni}_{0.1} \mathrm{Cu}_{0.05} \mathrm{~S}_{1.0}$ \\
\hline \multirow[t]{4}{*}{ V2-04 } & chalcopyrite & 4 \\
\hline & pentlandite & 15 \\
\hline & pyrrhotite & 81 \\
\hline & Mss composition & $\mathrm{Fe}_{0.83} \mathrm{Ni}_{0.09} \mathrm{Cu}_{0.02} \mathrm{~S}_{1.0}$ \\
\hline \multirow[t]{4}{*}{ V2-09_inc_1 } & chalcopyrite & 6 \\
\hline & pentlandite & 15 \\
\hline & pyrrhotite & 78 \\
\hline & Mss composition & $\mathrm{Fe}_{0.82} \mathrm{Ni}_{0.09} \mathrm{Cu}_{0.03} \mathrm{~S}_{1.0}$ \\
\hline \multirow[t]{4}{*}{ V2-09_inc_2 } & chalcopyrite & 9 \\
\hline & pentlandite & 11 \\
\hline & pyrrhotite & 80 \\
\hline & Mss composition & $\mathrm{Fe}_{0.82} \mathrm{Ni}_{0.07} \mathrm{Cu}_{0.04} \mathrm{~S}_{1.0}$ \\
\hline
\end{tabular}




\begin{tabular}{|c|c|c|}
\hline \multirow[t]{4}{*}{ V2-11 } & chalcopyrite & 7 \\
\hline & pentlandite & 15 \\
\hline & pyrrhotite & 78 \\
\hline & Mss composition & $\mathrm{Fe}_{0.82} \mathrm{Ni}_{0.09} \mathrm{Cu}_{0.03} \mathrm{~S}_{1.0}$ \\
\hline \multirow[t]{4}{*}{ V2-17 } & chalcopyrite & 4 \\
\hline & pentlandite & 16 \\
\hline & pyrrhotite & 80 \\
\hline & Mss composition & $\mathrm{Fe}_{0.82} \mathrm{Ni}_{0.10} \mathrm{Cu}_{0.02} \mathrm{~S}_{1.0}$ \\
\hline \multirow[t]{4}{*}{ V2-18_inc_1 } & chalcopyrite & 3 \\
\hline & pentlandite & 12 \\
\hline & pyrrhotite & 85 \\
\hline & Mss composition & $\mathrm{Fe}_{0.84} \mathrm{Ni}_{0.08} \mathrm{Cu}_{0.01} \mathrm{~S}_{1.0}$ \\
\hline \multirow[t]{4}{*}{ V2-18_inc_2 } & chalcopyrite & 8 \\
\hline & pentlandite & 17 \\
\hline & pyrrhotite & 75 \\
\hline & Mss composition & $\mathrm{Fe}_{0.80} \mathrm{Ni}_{0.11} \mathrm{Cu}_{0.04} \mathrm{~S}_{1.0}$ \\
\hline \multirow[t]{4}{*}{ V2-18_inc_3 } & chalcopyrite & 5 \\
\hline & pentlandite & 18 \\
\hline & pyrrhotite & 77 \\
\hline & Mss composition & $\mathrm{Fe}_{0.81} \mathrm{Ni}_{0.11} \mathrm{Cu}_{0.02} \mathrm{~S}_{1.0}$ \\
\hline \multirow[t]{4}{*}{ V2-19_inc_1 } & chalcopyrite & 5 \\
\hline & pentlandite & 17 \\
\hline & pyrrhotite & 79 \\
\hline & Mss composition & $\mathrm{Fe}_{0.82} \mathrm{Ni}_{0.10} \mathrm{Cu}_{0.02} \mathrm{~S}_{1.0}$ \\
\hline \multirow[t]{4}{*}{ V2-19_inc_2 } & chalcopyrite & 3 \\
\hline & pentlandite & 19 \\
\hline & pyrrhotite & 78 \\
\hline & Mss composition & $\mathrm{Fe}_{0.82} \mathrm{Ni}_{0.12} \mathrm{Cu}_{0.01} \mathrm{~S}_{1.0}$ \\
\hline \multirow[t]{4}{*}{ V2-19_inc_3 } & chalcopyrite & 7 \\
\hline & pentlandite & 20 \\
\hline & pyrrhotite & 73 \\
\hline & Mss composition & $\mathrm{Fe}_{0.80} \mathrm{Ni}_{0.12} \mathrm{Cu}_{0.03} \mathrm{~S}_{1.0}$ \\
\hline \multirow[t]{4}{*}{ V2-19_inc_4 } & chalcopyrite & 2 \\
\hline & pentlandite & 11 \\
\hline & pyrrhotite & 87 \\
\hline & Mss composition & $\mathrm{Fe}_{0.85} \mathrm{Ni}_{0.07} \mathrm{Cu}_{0.01} \mathrm{~S}_{1.0}$ \\
\hline \multirow{2}{*}{ V2-20_inc_1 } & chalcopyrite & 6 \\
\hline & pentlandite & 14 \\
\hline
\end{tabular}




\begin{tabular}{lll} 
& pyrrhotite & 81 \\
& Mss composition & $\mathrm{Fe}_{0.82} \mathrm{Ni}_{0.08} \mathrm{Cu}_{0.03} \mathrm{~S}_{1.0}$ \\
V2-20_inc_2 & chalcopyrite & 6 \\
& pentlandite & 12 \\
& pyrrhotite & 83 \\
& Mss composition & $\mathrm{Fe}_{0.83} \mathrm{Ni}_{0.07} \mathrm{Cu}_{0.03} \mathrm{~S}_{1.0}$ \\
& & \\
V2-20_inc_3 & chalcopyrite & 8 \\
& pentlandite & 14 \\
& pyrrhotite & 78 \\
& Mss composition & $\mathrm{Fe}_{0.81} \mathrm{Ni}_{0.09} \mathrm{Cu}_{0.04} \mathrm{~S}_{1.0}$ \\
& & \\
V2-20_inc_4 & chalcopyrite & 2 \\
& pentlandite & 8 \\
& pyrrhotite & 90 \\
& Mss composition & $\mathrm{Fe}_{0.86} \mathrm{Ni}_{0.05} \mathrm{Cu}_{0.01} \mathrm{~S}_{1.0}$ \\
\hline
\end{tabular}

Note: Jericho diamonds (Jer-2_A,-5_A and -16_A) contained only one inclusion of pyrrhotite 
Table S2: lattice parameters of pyrrhotite single-crystal inclusions

\begin{tabular}{llll}
\hline sample & $a(\AA)$ & $c(\AA)$ & $V\left(\AA^{3}\right)$ \\
\hline V2-01_inc1 & $3.447(1)$ & $5.707(3)$ & $58.71(5)$ \\
V2-01_inc2 & $3.443(1)$ & $5.711(2)$ & $58.63(3)$ \\
V2-01_inc3 & $3.444(2)$ & $5.703(3)$ & $58.58(6)$ \\
V2-02_inc2 & $3.421(18)$ & $5.673(9)$ & $57.5(4)$ \\
V2-02_inc3 & $3.461(4)$ & $5.642(7)$ & $58.52(12)$ \\
V2-09_inc1 & $3.469(5)$ & $5.668(6)$ & $59.07(13)$ \\
V2-09_inc2 & $3.437(4)$ & $5.694(9)$ & $58.27(14)$ \\
V2-19_inc1 & $3.460(2)$ & $5.674(3)$ & $58.82(7)$ \\
V2-19_inc2 & $3.451(2)$ & $5.665(3)$ & $58.42(5)$ \\
V2-19_inc3 & $3.458(5)$ & $5.672(8)$ & $58.74(15)$ \\
V2-19_inc4 & $3.483(6)$ & $5.680(10)$ & $59.68(17)$ \\
V2-20_inc1 & $3.456(2)$ & $5.667(4)$ & $58.64(7)$ \\
V2-20_inc2 & $3.473(3)$ & $5.646(6)$ & $58.97(10)$ \\
V2-20_inc4 & $3.455(3)$ & $5.656(6)$ & $58.47(10)$ \\
Jer-16_A & $3.490(2)$ & $5.790(3)$ & $61.06(5)$ \\
Jer-2_A & $3.494(3)$ & $5.799(3)$ & $61.30(7)$ \\
Jer-5_A & $3.488(4)$ & $5.691(6)$ & $59.97(11)$ \\
\hline
\end{tabular}


Table S3: orientation matrices of both inclusions and diamond host determined by single-crystal Xray diffraction. The format is the same as the one used in OrientXplot. The data can be pasted in a file .txt and if renamed as a dat file it can be read by the software to reproduce the data.

Title Sample

LAUE HOST m3m

OMTYPE CRYSALIS

WAVE 0.71074

HOST diamond V2-01_inc1

$\begin{array}{lll}0.125766 & 0.089816 & 0.128287\end{array}$

$\begin{array}{lll}-0.120796 & -0.053673 & 0.148868\end{array}$

$0.101733-0.167939 \quad 0.019146$

INC pyrrhotite V2-01_inc1

Laue inc $6 / \mathrm{mmm}$

$\begin{array}{lll}0.075772 & 0.084828 & 0.114388\end{array}$

$\begin{array}{lll}0.162163 & -0.069719 & -0.006856\end{array}$

$\begin{array}{llll}0.155469 & 0.210373 & -0.048180\end{array}$

HOST diamond V2-01_inc2

$\begin{array}{lll}0.125766 & 0.089816 & 0.128287\end{array}$

$\begin{array}{lll}-0.120796 & -0.053673 & 0.148868\end{array}$

$\begin{array}{lll}0.101733 & -0.167939 & 0.019146\end{array}$

INC pyrrhotite V2-01_inc2

Laue inc $6 / \mathrm{mmm}$

$\begin{array}{lll}-0.074879 & -0.202181 & -0.063557\end{array}$

$\begin{array}{lll}-0.190800 & -0.108204 & 0.073680\end{array}$

$\begin{array}{llll}-0.120177 & 0.063114 & -0.077307\end{array}$

HOST diamond V2-01_inc3

$\begin{array}{lll}0.125766 & 0.089816 & 0.128287\end{array}$ 
$\begin{array}{lll}-0.120796 & -0.053673 & 0.148868\end{array}$

$\begin{array}{lll}0.101733 & -0.167939 & 0.019146\end{array}$

INC pyrrhotite V2-01_inc3

Laue inc $6 / \mathrm{mmm}$

$\begin{array}{lll}-0.137341 & -0.085862 & 0.101064\end{array}$

$\begin{array}{lll}-0.004883 & 0.202700 & 0.011075\end{array}$

$\begin{array}{lll}-0.193989 & -0.089779 & -0.071670\end{array}$

HOST diamond V2-02 inc2

$\begin{array}{lll}-0.149232 & -0.118053 & 0.016504\end{array}$

$\begin{array}{lll}0.082262 & -0.124033 & -0.118931\end{array}$

$\begin{array}{lll}0.083649 & -0.086128 & 0.148090\end{array}$

INC pyrrhotite V2-02_inc2

Laue inc $6 / \mathrm{mmm}$

$\begin{array}{lll}0.187278 & -0.032750 & 0.020210\end{array}$

$\begin{array}{llll}0.018010 & -0.027304 & -0.122332\end{array}$

$\begin{array}{llll}0.150408 & 0.234922 & -0.011532\end{array}$

HOST diamond V2-02_inc3

$\begin{array}{lll}0.025310 & -0.147176 & -0.136623\end{array}$

$\begin{array}{lll}-0.182968 & -0.063427 & 0.031730\end{array}$

$\begin{array}{llll}-0.066508 & 0.119536 & -0.143038\end{array}$

INC pyrrhotite V2-02 inc3

Laue inc $6 / \mathrm{mmm}$

$\begin{array}{lll}-0.085226 & -0.221737 & 0.040435\end{array}$

$\begin{array}{llll}-0.052439 & -0.079558 & -0.117987\end{array}$

$\begin{array}{llll}0.214756 & 0.022399 & -0.012760\end{array}$

HOST diamond V2-09_inc1

$\begin{array}{lll}-0.061516 & 0.111197 & 0.161468\end{array}$ 
$\begin{array}{llll}0.103591 & 0.149748 & -0.060685\end{array}$

$\begin{array}{lll}-0.157572 & 0.075191 & -0.092231\end{array}$

INC pyrrhotite V2-09_inc1

Laue inc 6/mmm

$\begin{array}{lll}-0.007298 & 0.172792 & -0.061627\end{array}$

$\begin{array}{lll}-0.236536 & -0.131966 & -0.009012\end{array}$

$\begin{array}{lll}-0.020228 & 0.091925 & 0.109131\end{array}$

HOST diamond V2-09_inc2

$\begin{array}{lll}-0.061516 & 0.111197 & 0.161468\end{array}$

$\begin{array}{llll}0.103591 & 0.149748 & -0.060685\end{array}$

$\begin{array}{llll}-0.157572 & 0.075191 & -0.092231\end{array}$

INC pyrrhotite V2-09_inc2

Laue inc $6 / \mathrm{mmm}$

$\begin{array}{lll}0.217253 & 0.184929 & 0.018864\end{array}$

$\begin{array}{llll}0.022291 & 0.042567 & -0.123035\end{array}$

$\begin{array}{lll}-0.093953 & 0.141560 & 0.013201\end{array}$

HOST diamond V2-19_inc1

$\begin{array}{lll}0.022233 & 0.173586 & 0.093936\end{array}$

$\begin{array}{lll}-0.185117 & -0.014336 & 0.060747\end{array}$

$\begin{array}{lll}0.068466 & -0.097013 & 0.158914\end{array}$

INC pyrrhotite V2-19_inc1

Laue inc $6 / \mathrm{mmm}$

$\begin{array}{lll}-0.122575 & -0.225567 & 0.033992\end{array}$

$\begin{array}{lll}0.086970 & -0.060811 & -0.098206\end{array}$

$\begin{array}{lll}0.180865 & 0.026933 & 0.071328\end{array}$

HOST diamond V2-19_inc2

$\begin{array}{lll}-0.063213 & -0.194893 & 0.001971\end{array}$ 
$\begin{array}{lll}0.138970 & -0.051913 & -0.127562\end{array}$

$\begin{array}{lll}0.125033 & -0.043863 & 0.145425\end{array}$

INC pyrrhotite V2-19_inc2

Laue inc $6 / \mathrm{mmm}$

$\begin{array}{llll}-0.183418 & 0.023939 & -0.037392\end{array}$

$\begin{array}{lll}-0.150099 & -0.201931 & 0.058229\end{array}$

$\begin{array}{lll}0.017899 & 0.121868 & 0.104180\end{array}$

HOST diamond V2-19_inc3

$\begin{array}{llll}0.106939 & -0.136510 & -0.086221\end{array}$

$\begin{array}{lll}0.117661 & 0.148852 & -0.055273\end{array}$

$\begin{array}{lll}0.100493 & -0.029141 & 0.169869\end{array}$

INC pyrrhotite V2-19_inc3

Laue inc $6 / \mathrm{mmm}$

$\begin{array}{llll}0.163126 & -0.061183 & -0.028142\end{array}$

$\begin{array}{llll}0.163086 & 0.228681 & -0.012220\end{array}$

$\begin{array}{lll}0.054030 & 0.009210 & 0.120794\end{array}$

HOST diamond V2-19_inc4

$\begin{array}{lll}-0.107718 & -0.153476 & 0.035829\end{array}$

$\begin{array}{llll}0.103058 & -0.107370 & -0.119526\end{array}$

$\begin{array}{lll}0.115501 & -0.050574 & 0.145025\end{array}$

INC pyrrhotite V2-19_inc4

Laue inc 6/mmm

$\begin{array}{lll}-0.158277 & -0.201688 & 0.049652\end{array}$

$\begin{array}{llll}-0.172640 & 0.020562 & -0.055849\end{array}$

$\begin{array}{llll}0.015788 & -0.114158 & -0.100596\end{array}$

HOST diamond V2-20_inc1

$\begin{array}{lll}-0.176373 & 0.092262 & 0.030625\end{array}$ 
$\begin{array}{lll}-0.100304 & -0.137185 & -0.104560 \\ -0.029120 & -0.105082 & 0.153345\end{array}$

INC pyrrhotite V2-20_inc1

Laue inc $6 / \mathrm{mmm}$

$\begin{array}{lll}-0.177010 & -0.179575 & 0.060986\end{array}$

$\begin{array}{lll}-0.133143 & -0.062178 & -0.103988\end{array}$

$\begin{array}{lll}0.084982 & -0.141210 & -0.033379\end{array}$

HOST diamond V2-20_inc2

$\begin{array}{lll}0.055623 & -0.166665 & 0.090305\end{array}$

$\begin{array}{lll}-0.187117 & -0.064626 & -0.006870\end{array}$

$\begin{array}{llll}0.035804 & -0.082627 & -0.179918\end{array}$

INC pyrrhotite V2-20_inc2

Laue inc $6 / \mathrm{mmm}$

$\begin{array}{lll}-0.001539 & -0.176757 & 0.060754\end{array}$

$\begin{array}{lll}0.072236 & -0.065002 & -0.103363\end{array}$

$\begin{array}{lll}0.225771 & 0.140678 & 0.034196\end{array}$

HOST diamond V2-20_inc4

$\begin{array}{lll}-0.011005 & 0.184453 & 0.035479\end{array}$

$\begin{array}{llll}0.186432 & 0.029015 & -0.066632\end{array}$

$\begin{array}{llll}-0.066470 & 0.027678 & -0.191254\end{array}$

INC pyrrhotite V2-20_inc4

Laue inc $6 / \mathrm{mmm}$

$\begin{array}{lll}-0.149291 & -0.225067 & -0.031470\end{array}$

$\begin{array}{llll}0.089315 & 0.043086 & -0.116008\end{array}$

$\begin{array}{lll}0.161144 & -0.060013 & 0.034329\end{array}$

HOST diamond Jer-2_A

$\begin{array}{llll}0.114694 & -0.105018 & -0.122634\end{array}$ 
$\begin{array}{llll}0.081524 & 0.166469 & -0.066636\end{array}$

$\begin{array}{lll}0.138205 & -0.011240 & 0.140322\end{array}$

INC pyrrhotite Jer-2_A

Laue inc 6/mmm

$\begin{array}{lll}-0.054467 & -0.211535 & 0.041823\end{array}$

$\begin{array}{llll}0.048435 & -0.055260 & -0.109616\end{array}$

$\begin{array}{lll}0.223124 & 0.083746 & 0.034489\end{array}$

HOST diamond Jer-5_A

$\begin{array}{lll}-0.063836 & -0.182233 & 0.035160\end{array}$

$\begin{array}{llll}0.130223 & -0.071709 & -0.128562\end{array}$

$\begin{array}{lll}0.132233 & -0.019375 & 0.144483\end{array}$

INC pyrrhotite Jer-5_A

Laue inc $6 / \mathrm{mmm}$

$\begin{array}{llll}0.100115 & -0.114638 & -0.051041\end{array}$

$\begin{array}{lll}-0.184453 & -0.204067 & 0.034033\end{array}$

$\begin{array}{lll}-0.104850 & -0.010683 & -0.108215\end{array}$

HOST diamond Jer-16_A

$\begin{array}{llll}0.029389 & -0.159505 & -0.112573\end{array}$

$\begin{array}{llll}0.191947 & 0.041151 & -0.014367\end{array}$

$\begin{array}{lll}0.036645 & -0.109170 & 0.160304\end{array}$

INC pyrrhotite Jer-16 A

Laue inc $6 / \mathrm{mmm}$

$\begin{array}{lll}-0.035065 & 0.149944 & -0.067008\end{array}$

$\begin{array}{lll}-0.208470 & -0.077124 & 0.053721\end{array}$

$\begin{array}{lll}0.101093 & 0.162701 & 0.087623\end{array}$ 